\title{
Coupling between tolerance and resistance differs between related Eimeria parasite species: implications for co-evolution with their mouse hosts
}

\author{
Alice Balard ${ }^{1}$, Víctor Hugo Jarquín-Díaz ${ }^{1}$, Jenny Jost ${ }^{1}$, Vivian Mittné ${ }^{1}$, Francisca \\ Böhning $^{1}$, Ľudovít Ďureje ${ }^{2}$, Jaroslav Piálek ${ }^{2}$, and Emanuel Heitlinger ${ }^{1}$ \\ ${ }^{1}$ Humboldt University of Berlin Institute of Biology \\ ${ }^{2}$ Institute of Vertebrate Biology Czech Academy of Sciences
}

June 29, 2020

\begin{abstract}
Resistance (host capacity to reduce parasite burden) and tolerance (host capacity to reduce impact on its health for a given parasite burden) manifest two different lines of defence. Tolerance can be independent from resistance, traded-off against it, or the two can be positively correlated because of redundancy in underlying (immune) processes. We here tested whether this coupling between tolerance and resistance could differ upon infection with closely related parasite species. We tested this in experimental infections with two parasite species of genus Eimeria. We measured proxies for resistance (the (inverse of) number of parasite transmission stages (oocysts) per gram of feces at the day of maximal shedding) and tolerance (the slope of maximum relative weight loss compared to day of infection on number of oocysts per gram of feces at the day of maximal shedding for each host strain) in four inbred mouse strains and four groups of F1 hybrids belonging to two mouse subspecies, Mus musculus domesticus and M. m. musculus. We found a negative correlation between resistance and tolerance against E. falciformis, while the two are uncoupled against E. ferrisi. We conclude that resistance and tolerance against the first parasite species might be traded off, but evolve more independently in different mouse genotypes against the latter. We argue that evolution of the host immune defences can be studied largely irrespective of parasite isolates if resistance-tolerance coupling is absent or weak (E. ferrisi) but host-parasite coevolution is more likely observable and best studied in a system with negatively correlated tolerance and resistance (E. falciformis).
\end{abstract}

\section{Hosted file}

Article_main.pdf available at https://authorea.com/users/337944/articles/463612-couplingbetween-tolerance-and-resistance-differs-between-related-eimeria-parasite-speciesimplications-for-co-evolution-with-their-mouse-hosts 